\title{
BMJ Open Protocol for the impact of CBT for insomnia on pain symptoms and central sensitisation in fibromyalgia: a randomised controlled trial
}

\author{
Christina S McCrae (D) , ${ }^{1}$ Ashley F Curtis (D) , ${ }^{2}$ Jason Craggs, ${ }^{3}$ Chelsea Deroche,${ }^{4}$ \\ Pradeep Sahota, ${ }^{5}$ Chokkalingam Siva, ${ }^{6}$ Roland Staud, ${ }^{7}$ Michael Robinson ${ }^{8}$
}

To cite: McCrae CS, Curtis AF, Craggs J, et al. Protocol for the impact of CBT for insomnia on pain symptoms and central sensitisation in fibromyalgia: a randomised controlled trial. BMJ Open 2020;10:e033760. doi:10.1136/ bmjopen-2019-033760

- Prepublication history for this paper is available online. To view these files, please visit the journal online (http://dx.doi. org/10.1136/bmjopen-2019033760).

Received 20 August 2019 Revised 24 April 2020 Accepted 02 July 2020

Check for updates

(C) Author(s) (or their employer(s)) 2020. Re-use permitted under CC BY-NC. No commercial re-use. See rights and permissions. Published by BMJ.

For numbered affiliations see end of article.

Correspondence to Dr Christina S McCrae; mccraec@health.missouri.edu

\section{ABSTRACT}

Introduction Approximately $50 \%$ of individuals with fibromyalgia (a chronic widespread pain condition) have comorbid insomnia. Treatment for these comorbid cases typically target pain, but growing research supports direct interventions for insomnia (eg, cognitive behavioural treatment for insomnia (CBT-I)) in these patients. Previous research suggests sustained hyperarousal mediated by a neural central sensitisation mechanism may underlie insomnia and chronic pain symptoms in fibromyalgia. We hypothesise CBT-I will improve insomnia symptoms, improve clinical pain and reduce central sensitisation. The trial will be the first to evaluate the short-term and longterm neural mechanisms underlying insomnia and pain improvements in fibromyalgia. Knowledge obtained from this trial might allow us to develop new or modify current treatments to better target pain mechanisms, perhaps reversing chronic pain or preventing it.

Methods and analysis Female participants $(n=130)$ 18 years of age and older with comorbid fibromyalgia (with pain severity of at least 50/100) and insomnia will be recruited from the University of Missouri in Columbia, Missouri, and surrounding areas. Participants will be randomised to 8 weeks (plus 4 bimonthly booster sessions) of CBT-I or a sleep hygiene control group (SH). Participants will be assessed at baseline, post-treatment, 6 and 12 months follow-ups. The following assessments will be completed: 2 weeks of daily diaries measuring sleep and pain, daily actigraphy, insomnia severity index, pain-related disability, single night of polysomnography recording, arousal (heart rate variability, cognitive affective arousal), structural and functional MRI to examine painrelated neural activity and plasticity and mood (depression, anxiety).

Ethics and dissemination Ethics approval was obtained in July 2018 from the University of Missouri. All data are expected to be collected by 2022. Full trial results are planned to be published by 2024 . Secondary analyses of baseline data will be subsequently published.

Trial registration number NCT03744156.

\section{INTRODUCTION}

\section{Background}

Chronic pain imposes a substantial public health burden, afflicting over 100 million
Strengths and limitations of this study

- Eight-week cognitive behavioural therapy for insomnia (CBT-I) integrates sleep education, hygiene, stimulus control, sleep restriction, relaxation and cognitive restructuring techniques and will be examined relative to an active sleep hygiene (SH) control in patients with comorbid fibromyalgia and insomnia.

- Pain severity cut-off criteria maximises potential to observe clinical and neural pain-related improvements following CBT-I.

- Investigation of neural pain mechanisms underlying effects of CBT-I relative to an active control will further understanding of central mechanisms underlying change in pain or insomnia in fibromyalgia, which may inform mechanisms underlying sustained pain in fibromyalgia.

- Six and 12 months follow-ups will enable examination of persistence of behavioural and neural outcomes of CBT-I.

- Potential limitations include participant attrition at follow-up, which may contribute to selection bias associated with systematic differences between participants completing CBT-I versus SH. Additionally, there is no healthy control comparison group, thereby we will be unable to determine whether neural mechanisms of chronic pain are specific to patients with fibromyalgia or generalisable to the broader population.

Americans, with annual costs estimated at over US $\$ 500$ billion. ${ }^{1}$ Individuals with chronic pain consume more healthcare services, yet $40 \%$ report inadequate management of their pain. ${ }^{2}$ Chronic insomnia (at least 3 months of difficulty initiating and/or maintaining sleep or early morning awakening, accompanied by dysfunction in at least one area of daytime functioning such as social, occupational, educational, academic, behavioural, etc) ${ }^{3}$ is highly comorbid with pain, affecting at least $50 \%$ of patients with chronic pain. ${ }^{4}$ Recent 
research suggests chronic insomnia can lead to the development or worsening of chronic pain. ${ }^{5}$ Thus, research efforts to prevent, and treat chronic pain should consider sleep disturbance as a primary intervention target. ${ }^{5}$

The relationship between fibromyalgia (a chronic condition characterised by widespread pain) and sleep disturbance is well established. ${ }^{6}$ Research on fibromyalgia has linked reports of restful sleep with less reported discomfort and fatigue, ${ }^{7}$ and non-restorative sleep with exacerbation of pain. ${ }^{8}$ Polysomnographic studies have identified sleep architecture differences in patients with fibromyalgia versus healthy controls (ie, increased sleep onset latency (SOL) ${ }^{9}$ lighter sleep, ${ }^{910}$ more arousals, ${ }^{11-13}$ reduced deep sleep. ${ }^{911}{ }^{12}$ More than $50 \%$ of persons with fibromyalgia meet insomnia criteria, ${ }^{14} 15$ and fibromyalgia diagnostic criteria were recently revised to include sleep disturbance as a core feature. ${ }^{16}$ The causal role of sleep in the aetiology of chronic pain has gained empirical support. ${ }^{17} 18$ Longitudinal, experimental and trial evidence over the past decade suggest sleep may be a more reliable predictor of chronic pain, than vice versa. ${ }^{519}$ When chronic pain and insomnia co-occur, treatment typically focuses on pain. The insomnia is considered a symptom and thus, is expected to improve following improvement in pain. However, a growing body of research, ${ }^{5}$ including our recent trial, ${ }^{20}$ supports direct intervention for insomnia in the context of pain.

Fibromyalgia is characterised by chronic widespread pain, central sensitisation (CS) and mechanical allodynia. ${ }^{15}$ The predominant pathophysiology of pain in fibromyalgia is abnormal central pain processing or CS. ${ }^{15} \mathrm{CS}$ is characterised by increased responsiveness of the central nervous system (CNS) to noxious and nonnoxious stimuli. Hyperalgesia and allodynia, important consequences of CS, are related to increased excitability of spinal and supraspinal neurons. ${ }^{21}$ Patients with fibromyalgia have a higher rate of temporal summation of heatevoked second pain (TSSP), a proxy for CS, compared with pain-free controls. ${ }^{21}$ TSSP (aka wind-up) and subsequent aftersensations are greatly prolonged in fibromyalgia. ${ }^{21}$ Importantly, mechanical allodynia, enhanced wind-up and prolonged aftersensations represent CS features found to be relevant predictors of fibromyalgia clinical pain. $^{22}$

The Cognitive Activation Theory of Stress (CATS) posits chronic arousal leads to changes in the CNS consistent with $\mathrm{CS} .^{23}$ CATS provides a framework illustrating the mechanisms by which CBT-I can improve pain. CATS proposes that through chronic arousal and insomnia (which has been linked to arousal-as described below), there are critical changes to hypothalamic-pituitary-adrenal (HPA) and CNS functioning that prompt increased sensitivity to stimulation, particularly pain. ${ }^{23}$ We propose CBT-I improves pain by reducing arousal and improving sleep; thereby, reversing the negative HPA and CNS changes (ie, reversing CS) that sustain chronic pain. Cognitive factors are key contributors to arousal in CATS and have a strong empirical basis to support their relationship to insomnia and chronic pain. ${ }^{724}$ Chronic arousal and poor sleep, via their effects on the nervous system, are plausible candidates for explaining the relationships of cognitive states (catastrophising, somatic focus) and ongoing nociceptive input ${ }^{8}$ to $\mathrm{CS}$ and chronic pain.

Hyperarousal is a well-established maintenance factor of chronic insomnia. ${ }^{25}$ Persons with insomnia often develop increased cognitive focus and catastrophising cognitions (eg, "I will never sleep well again") that increase arousal and interfere with getting good sleep. Cognitive therapy (a component of CBT-I) effectively targets and replaces such thoughts (eg, 'Everyone sleeps poorly on occasion'); thereby, reducing cognitive arousal and improving sleep. Previously, we found CBT-I produced large, significant improvements in sleep-related and pain-related cognitiveaffective arousal. ${ }^{20}$ However, because arousal is a multidimensional construct, we have included multiple measures in the present trial-cognitive (non-specific (perceived stress), condition specific (dysfunctional sleep cognitions, pain catastrophising)) and peripheral (heart rate variability (HRV)). In terms of peripheral arousal, studies have found alterations in heart rate (HR) and HRV while awake before sleep and during stage 2 non-rapid eye movement sleep, ${ }^{26}$ increased low frequency power and decreased high frequency power across all sleep stages ${ }^{27}$ and lower wake-to-sleep HR reduction and SD of N-N intervals, ${ }^{28}$ in persons with chronic insomnia compared with controls, consistent with increased sympathetic activity. An uncontrolled study in patients with primary insomnia found alterations of HRV following CBT-I. ${ }^{29}$ Given our theoretical framework that CBT-I will prompt a reduction of arousal, we expect a decrease of sympathetic activity (ie, increase in HRV) at post-treatment and both follow-ups in the proposed study.

Pain is multidimensional and evidence indicates different brain regions, ${ }^{30}$ CNS pathways ${ }^{31}$ and functional interactions ${ }^{32}$ are dynamically involved in creating the subjective pain experience. Research has identified changes in neural activity in a variety of brain regions that are positively correlated with pain. ${ }^{33}$ These regions comprise various neural networks involved with processing different dimensions of the pain experience: parts of medial and posterior thalamus, somatosensory cortices (S1/S2), insular cortical areas (posterior, mid, anterior insula), cingulate cortical areas (subgenual and pregenual anterior cingulate, anterior midcingulate, posterior cingulate), caudate/putamen and cerebellar areas. ${ }^{35-37}$ The purpose of neuroimaging in the current trial is to increase knowledge of the underlying neural mechanisms associated with chronic pain and how to manipulate them to treat chronic pain by targeting insomnia. Consistent with CATS,${ }^{38}$ our functional MRI (fMRI) results suggest chronic pain may result from abnormal pain modulation processes. In our previous imaging work, results indicated aberrant pain processing seen in fibromyalgia is due to sustained arousal across common pain processing networks. ${ }^{39} 40$ Moreover, we identified treatment-related changes in neural activity 
among brain regions involved in the cognitive and affective dimensions of pain. ${ }^{32} 41$

Fibromyalgia is associated with grey matter atrophy in the amygdala, cingulate, insula, medial frontal cortex, parahippocampus and prefrontal cortex. ${ }^{182-44}$ Neuroimaging research has also associated chronic insomnia with reduced grey matter in the amygdala, orbitofrontal cortex and precuneus. ${ }^{45}{ }^{46}$ Of particular interest, several studies have examined the Default Mode Network (DMN) and found both fibromyalgia ${ }^{47}$ and insomnia ${ }^{49} 50$ are characterised by heightened activity and connectivity patterns not typically observed in healthy persons. The present trial will be the first to examine CBT-I's impact on the DMN in patients with fibromyalgia and comorbid insomnia.

The efficacy of CBT-I is well established. The vast majority $(\sim 70 \%-80 \%)$ of persons with insomnia treated behaviourally show sleep improvements that maintain through follow-ups up to 2 years, and patients rate behavioural techniques as more acceptable than sleep medications. ${ }^{17}$ Unlike sleep medications, behavioural approaches do not pose serious side effects and may be more cost-effective in the long run. ${ }^{51} \mathrm{~A}$ meta-analysis ${ }^{52}$ of non-pharmacological interventions for insomnia (all involved at least one component of CBT-I) in patients with chronic pain (11 RCTs, 3 involving fibromyalgia) found large sleep quality improvements and small-to-moderate pain reductions following treatment. Sleep quality effects were maintained at 1 year. To date, most CBT-I trials in patients with chronic pain, ${ }^{52}$ and the two CBT-I trials in fibromyalgia ${ }^{53} 54$ have not required a specific minimum level of pain severity for inclusion. Thus, it is possible that individuals with low levels of pain were included in these trials and did not have high enough initial pain severity to show substantial improvement.

The proposed trial examines the novel hypothesis that sustained improvements in arousal, sleep and CS will result in sustained (or possibly enhanced) pain improvements over time. Currently, the long-term effects of CBT-I on clinical pain and its underlying neural mechanisms in fibromyalgia are unknown. The proposed trial offers the following methodological improvements: (1) recruitment of participants with more severe baseline pain, (2) expanded arousal outcomes (peripheral arousal, global cognitive arousal/stress, sleep-related and pain-related cognitive-affective factors), (3) imaging follow-ups at 6 and 12 months, (4) booster sessions (to ensure longterm maintenance of treatment effects), (5) a credible active control-sleep hygiene ( $\mathrm{SH}$ ) (to control for attentional/non-specific therapeutic effects) and (6) inclusion of moderation/mediation analyses. The proposed trial addresses key shortcomings in our current understanding of chronic pain.

\section{Aims}

The overarching goal of this randomised controlled trial is to study effects of CBT-I on objective and subjective measures of sleep, arousal and pain, as well as examine the temporal relationships between our hypothesised mediators (sleep and arousal) and pain. In our recent trial, CBT-I prompted larger initial improvements in sleep $^{20}$ and $\mathrm{CS}^{55}$ than did cognitive behavioural treatment for pain. Given sleep and CS's hypothesised mediating roles, we focus on CBT-I only here as the intervention. Additionally, given potential impact of non-specific therapeutic factors on outcomes, we compare CBT-I with an active and credible control condition, $\mathrm{SH}$.

Our first specific aim is to examine the effects of 8 weeks of CBT-I relative to 8 weeks of SH control on arousal (HRV, cognitive-affective-dysfunctional sleep and pain cognitions, perceived global stress), subjective/objective sleep (SOL, wake after sleep onset (WASO), sleep efficiency and quality; insomnia impact) and pain after treatment and at 6 and 12 months follow-ups. We hypothesise that compared with SH, CBT-I will decrease arousal, improve sleep and decrease pain after treatment and at 6 and 12 months follow-ups. Our second specific aim is to examine CBT-I's effect on resting state (RS) brain activity as well as neural activation patterns of functional brain networks and blood-oxygen-level dependent (BOLD) responses to painful stimuli in regions associated with pain processing. We hypothesise that compared with $\mathrm{SH}$, CBT-I will reduce (normalise) RS brain activity in the DMN, which includes the cingulate cortex and medial prefrontal cortex, and reduce maladaptive pain-related brain network and BOLD activity in several regions associated with the cognitive and affective modulation of pain, including the inferior frontal gyrus, cingulate gyrus and insula. Our third specific aim is to study CBT-I's long-term effect on structural characteristics of pain-related brain regions. We hypothesise that compared with SH, CBT-I will prompt structural changes indicative of a reversal of the maladaptive neural plasticity associated with chronic pain. Reversal will be characterised by increased grey matter volume/thickness, improved white matter integrity and stronger structural connectivity in the lateralorbitofrontal and anterior/rostral cingulate regions, compared with the control following treatment and at both follow-ups. Finally, our fourth aim is to examine the mediating impact of arousal, sleep and CS on pain. We hypothesise that CBT-I will promote pain improvements through arousal reduction, sleep improvement and CS reversal. We hypothesise that significant improvements in all variables will be evident immediately following treatment, and that sustained improvements in arousal, sleep and CS will mediate sustained (and possibly increased) improvements in pain at 6 and 12 months. We will also evaluate whether these mediating effects explain unique variance of pain improvement over and beyond the mediating effects of global or possibly pain-specific and/or sleep-specific cognitive-affective factors.

\section{METHODS}

\section{Trial design and study setting}

Female patients (18 years of age and older) with fibromyalgia and chronic insomnia will be recruited from 


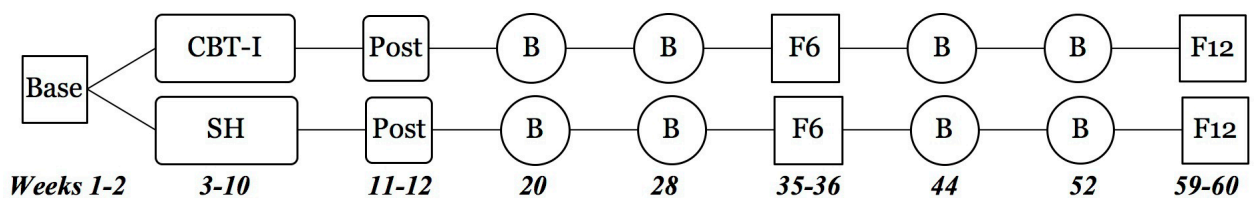

Figure 1 Timeline of randomised controlled trial. CBT-I, cognitive behavioural treatment for insomnia; SH, sleep hygiene.

the University of Missouri in Columbia, Missouri, and surrounding area. Participants will be recruited through physician referral from Rheumatology, Internal Medicine and Sleep Clinics as well as community advertisements. Participants will be randomised to 8 weeks of CBT-I or SH. Both groups will receive four bimonthly phone booster sessions (B; figure 1). Baseline, post-treatment and 6 and 12 months follow-ups will measure sleep, arousal, neural plasticity and pain. All participants will sign written informed consent. Participants will be compensated US $\$ 150$ following the baseline, post-treatment, 6 and 12 months follow-up assessments.

\section{Eligibility criteria}

Inclusion criteria are: (1) female, (2) 18+ years of age, (3) willing to be randomised, (4) can read and understand English, (5) diagnosed with fibromyalgia ((a) pain for $6+$ months that is (b) confirmed by tender point test (with application of $4 \mathrm{~kg}$ force, participant reports pain in at least 11 of 18 points, including points in all four body quadrants ${ }^{15}$ ) and (c) baseline diaries indicate average pain intensity of $\geq 50 / 100)$ and insomnia ((a) insomnia complaints for $6+$ months that (b) occur despite adequate opportunity and circumstances for sleep, (c) consist of one or more of the following: difficulty falling asleep, staying asleep or waking up too early, (d) daytime dysfunction (mood, cognitive, social, occupational) due to insomnia and (e) baseline diaries indicate $>30$ min of SOL or WASO on six or more nights), (6) no prescribed or over-the-counter pain or sleep medications for $1+$ month, or stabilised on medications for $6+$ weeks.

Exclusion criteria are: (1) unable to provide informed consent, (2) cognitive impairment (Mini-Mental State Examination $<26)$, (3) sleep disorder other than insomnia (ie, sleep apnoea (apnoea/hypopnea index $>15$ ), periodic limb movement disorder (myoclonus arousals per hour $>15)$ ), (4) bipolar or seizure disorder (due to risk of sleep restriction treatment), (5) other major psychopathology except depression or anxiety (eg, suicidal ideation/ intent, psychotic disorders), (6) severe untreated psychiatric comorbidity (eg, schizophrenia, substance use disorder), (7) psychotropic or other medications (eg, beta-blockers) that alter pain or sleep, (8) participation in non-pharmacological treatment (including CBT) for pain, sleep or mood outside current trial, (9) internal metal objects or electrical devices and (10) pregnancy.

\section{Randomisation}

Biostatistician (CD) will select block size and perform randomisation. Other personnel (except for therapists and project coordinator) will be blinded to randomisation.
Blocking guarantees balance, increases power ${ }^{56}$ and will be accounted for in analyses.

\section{Procedures}

\section{Screening}

Screening to assess fibromyalgia and insomnia symptoms and to rule out sleep disorders other than insomnia is carried out in four stages:

Stage 1: brief screener $(\sim 10 \mathrm{~min})$. The project coordinator will conduct a brief structured interview to address inclusion/exclusion criteria and establish probable fibromyalgia and insomnia diagnoses.

Stage 2: clinical interview ( 50 min). The assessor will: (1) conduct a semi-structured pain, sleep and psychiatric in-person interview, (2) perform tender point testing. Diagnosis of fibromyalgia will be overseen by a rheumatologist (CS).

Stage 3: polysomnography (PSG; one overnight). One night of polysomnography will rule out sleep disorders other than insomnia (ie, apnoea, PLMD). The assessor will prepare participants for PSG in their own homes, and participants will sleep in their own beds. Referrals will be made for those disqualified to a neurologist (PS).

Stage 4: sleep diary confirmation of insomnia $(\sim 5$ $\mathrm{min}$ /day). Baseline sleep diaries will be used to confirm insomnia diagnosis and must show: $>30 \mathrm{~min}$ of SOL or WASO on $6+$ nights during the 2 weeks. Sleep diaries will be collected electronically via an online data management system (Qualtrics) with personal web-enabled devices or (if needed) study provided devices. Diagnosis of insomnia will be overseen by a sleep psychologist (CSMcC) .

\section{Interventions}

Both interventions include 8 weekly, 50 min individual face-to-face sessions with a therapist (predoctoral graduate students in an APA accredited clinical or school psychology programme at the University of Missouri) and 4 bimonthly, 20 min phone booster sessions. SH will meet on the same schedule as CBT-I, controlling for therapist attention and other non-specific therapeutic factors. Session content for CBT-I and SH are provided in tables 1 and 2 , respectively.

\section{Treatment integrity}

The three-step method by Lichstein $e t a \tilde{l}^{7}$ will be used to measure treatment integrity.

\section{Treatment delivery/training}

Therapists will use manuals. Practice will begin with mock sessions and then recorded sessions with volunteers. The Principal Investigator (PI; CSMcC) will score all training 
Table 1 Session content for CBT-I

Session number Content

1. Sleep education Participants will be provided with education on sleep stages; sleep and fibromyalgia and circadian rhythms and sleep. This information is given to provide a heuristic background for the specific sleep techniques used.

2. Sleep hygiene $(\mathrm{SH})$

$\mathrm{SH}$ will be discussed and participants are instructed to adhere to the following rules: (1) avoid caffeine after noon, (2) within 2 hours of bed, avoid exercise, nicotine, alcohol and heavy meals, (3) within 1 hour of bedtime, avoid screen time. The goal of $\mathrm{SH}$ is to eliminate sleepinterfering behaviours.

3. Stimulus control (SC) and brief relaxation

SC will be discussed and participants will be asked to adhere to the following recommendations: (1) do not use bed/bedroom for anything but sleep (or sex), (2) if not asleep in 15-20 min, leave bed, do something non-arousing in another room. Return to bed when sleepy. If not asleep in 20 min, repeat., (3) if awake and not back asleep in 20 min, repeat \#2, (4) avoid napping. The goal of SC is to break incompatible/build compatible sleep associations. In addition, a 10 min relaxation exercise will be recorded and given to participants for practice at bedtime and once during the day. The goal of this is to induce relaxation/reduce arousal.

4. Sleep restriction

A time in bed prescription $(\mathrm{Rx})$ will be set at baseline average diary reported total sleep time plus $30 \mathrm{~min}$. If this value is $<5$ hours, $\mathrm{Rx}$ will be set at 5 hours. The therapist and participant will work together to set regular bed/wake times consistent with Rx. The goal of sleep restriction is to regulate sleep-wake cycle and reduce awake time in bed.

\section{Monitoring automatic} thoughts

Thoughts, thought patterns and emotional reactions that interfere with getting good sleep (ie, "I will never sleep well again".) will be identified and monitored.
The validity of sleep-interfering thoughts will be challenged and replaced with sleep conducive ones (ie, "There are things I can do to improve my sleep".)
6. Challenging/Replacing

dysfunctional thoughts conducive ones (ie, "There are things l can do to improve my sleep".)

\section{Practical recommendations}

8. Review and maintenance

Booster sessions
Established cognitive restructuring techniques (ie, reappraisal, reattribution and decatastrophising) will be taught.

Learnt skills and importance of a regular sleep schedule and good sleep habits will be reviewed. Continued use of the techniques learnt will be discussed.

In this brief ( 20 min) telephone session, techniques from sessions 1 to 8 will be reviewed. The therapist will encourage continued practice of techniques. Problems will be troubleshooted.

CBT-I, cognitive behavioural treatment for insomnia.

sessions. Training will last $\sim 16$ weeks until therapists obtain mastery (scoring 100 on each session's Treatment Delivery Score Sheet). For assessment of participant treatment delivery, all sessions will be recorded. Fifty per cent will be scored by consultant (registered psychologist). Senior consultant (registered psychologist) will double score initial 10 treatment and 10 booster sessions to establish fidelity, and $10 \%$ of remaining sessions for reliability. Consultants will inform the PI of scores $<95 \%$ for supervisory/training purposes. The PI will review $25 \%$ and therapists will review $25 \%$ of each other's sessions for ongoing training/supervision. Only consultant reviews will be used to assess fidelity.

\section{Treatment receipt}

To ensure treatment comprehension, participants will be encouraged to ask questions. Workbooks describe and reinforce treatment content. To assess treatment receipt, participants will complete a brief quiz at end of session 4 .

\section{Treatment enactment}

To ensure home assignments are done, workbooks contain written instructions on home assignments. To assess enactment, participants will maintain daily electronic diaries and logs.

\section{Treatment credibility and expectancy}

At the end of session 3, participants will complete a treatment credibility questionnaire. This 4-item scale assesses the participant reaction to therapist and treatment efficacy, and participants provide ratings of 1 (strongly disagree) to 10 (strongly agree). Higher scores represent better treatment credibility. At the end of session 3 therapists will complete an expectancy for improvement scale.

\section{Outcomes}

A summary of study outcomes is provided in table 3 and a schedule of outcome measures is provided in table 4 . A full description of the thermal pain task conducted 
Table 2 Session content for sleep hygiene education

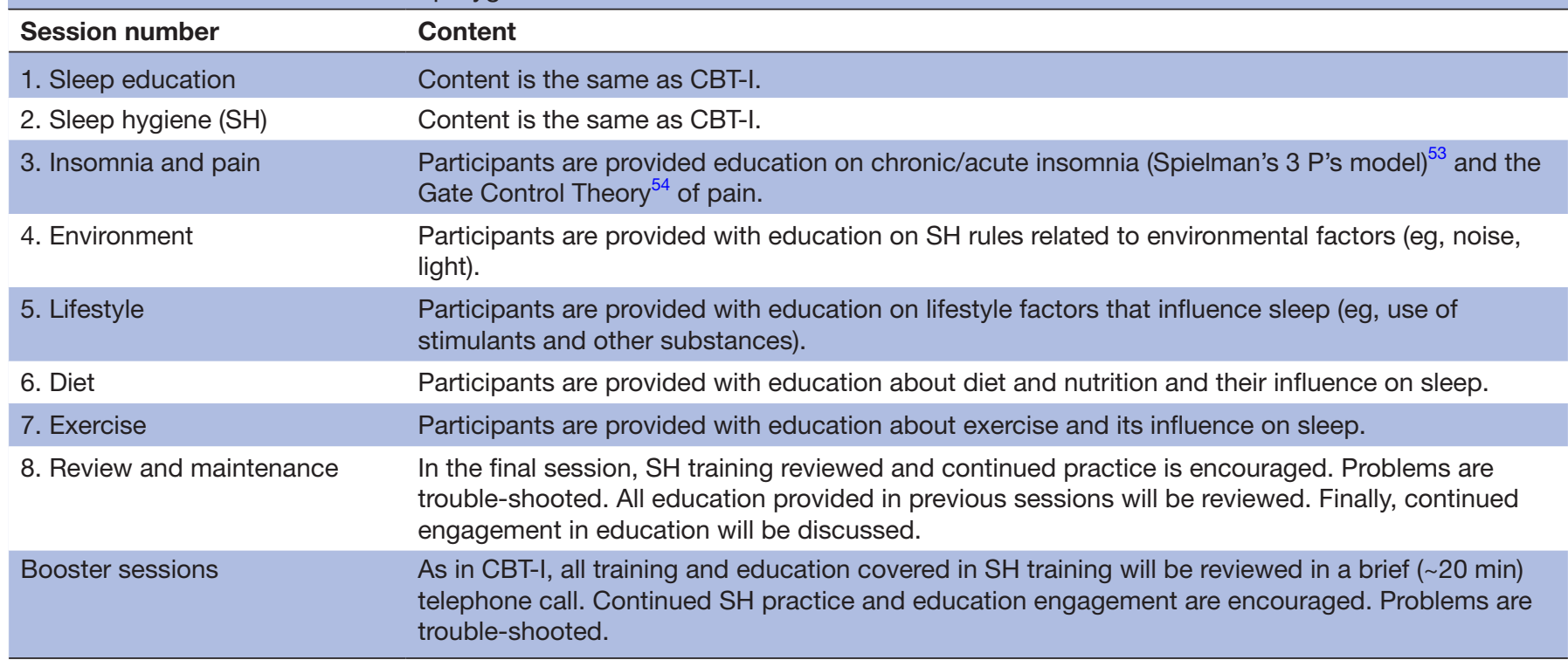

CBT-I, cognitive behavioural treatment for insomnia.

during fMRI scanning is provided in table 3. Briefly, for this task, the thermal stimuli will be delivered with an MR compatible, computer-controlled, CHEPS Pathway system, which is a peltier-element-based stimulator, which is capable of producing stimuli across a range of temperatures $\left(33^{\circ} \mathrm{C}-51^{\circ} \mathrm{C}\right)$. The start of each scan will begin with the thermode on the left foot at ambient temperature for $42 \mathrm{~s}$ and then 16 cycles of the following: the pain temperature (determined by the calibration trials), and remain at that temperature (hold) for $5 \mathrm{~s}$, followed by a variable interstimulus interval with an average between 10 and 12 s. Following the 16th cycle, the scan proceeds for another $30 \mathrm{~s}$ with the thermode at ambient temperature. After each scan, participants will report the average and max pain rating during the scan.

\section{Study timeline}

The study timeline is provided in table 5 .

\section{Analytical approach \\ Power analysis}

Effect sizes in our prior trial that were small for pain $(f=0.2)$, medium to large for sleep $(f=0.31-39)$, large for imaging $(f=0.69-1.13)$, and large for pain-related and sleep-related cognitive-affective arousal $(f=0.69-1.13)$. To ensure adequate power with an active control, effect sizes from CBT-I trials in fibromyalgia with SH control groups were considered and ranged from small to medium for pain $(f=0.15-0.25$; including pain-related anxiety) and small to large for sleep outcomes $(f=0.15-0.40) .{ }^{3}{ }^{4}$ Our prior trial did not measure peripheral arousal. However, based on prior research, ${ }^{7}$ small to medium effects $(f=0.15-0.25)$ are expected. We determined power based on the traditional repeated measures analysis of variance (RM ANOVA) approach, as there are no established procedures for accurate power estimation for multi-level modelling (MLM). Using G-Power ${ }^{58}$ for RM ANOVA within-between interaction, setting $\alpha=0.05$, number of groups $=2$, number of measurements $=4$ and correlations between repeated measures $=0.5$, minimum statistical power $=0.8$, the sample size required to detect a small effect of $f=0.15$ is 62 . For the mediation model tested in aim 4, given that the effect sizes (ESs) of the mediating paths range from small to large $(f=0.15-0.40)$, a sample size of 130 provides sufficient power $(>0.8)$ to detect the mediation effects on pain. ${ }^{59}$

\section{Missing values}

Missing data will also be accounted for using MLM. This statistical procedure can handle missing data at all levels except the highest, which in our case, is level 2. When collecting measurements from the same people over time, some may not complete the study. Unlike RM ANOVA which would exclude these participants' data from analysis, with MLM, their information is retained in the prediction model which increases statistical power. Additional steps will be followed: (1) group dropout rates will be compared using $\chi^{2}$ analyses, (2) demographic and dependent variables will be examined for relationship to dropout, using related variables to impute missing values in analyses below (via SPSS Missing Items Analysis), (3) comparison of completers versus imputated analyses to further estimate dropout effects.

\section{Baseline demographics and participant characteristics}

Group differences in baseline demographics and clinical characteristics will be analysed using independent sample t-tests for continuous variables (number of health conditions, body mass index, Mini-Mental State Examination, duration of fibromyalgia, duration of insomnia) and $\chi^{2}$ analyses for categorical variables (sex, marital status, ethnicity, employment status, sleep or pain medication 
Table 3 Outcome measures

\begin{tabular}{lll}
$\begin{array}{l}\text { Outcome } \\
\text { category }\end{array}$ & Measure & Primary/Secondary \\
\hline $\begin{array}{l}\text { Subjective } \\
\text { sleep }\end{array}$ & Daily sleep diaries & Primary \\
\hline
\end{tabular}

Insomnia Severity Index Primary (ISI)

Perimary

Details

Online diaries will be completed each morning ( $\sim \mathrm{min})$ during each 2 week assessment period and 8 weeks of treatment. Primary outcome variables include: sleep onset latency (time from initial lights-out until sleep onset), wake after sleep onset (time awake after initial sleep onset until last awakening), number of awakenings, total sleep time, sleep efficiency (total sleep time/time spent in bed $\times 100$ ) and sleep quality rating ( $1-$ very poor to $5-$ excellent). Sleep and pain medication consumption variables will include: name, dosage and time taken. Sleep medication will be converted to number of lowest recommended dosage units, ${ }^{72}$ and pain medication to morphine equivalent dosage. ${ }^{73}$

At each time point, participants will complete the ISI (primary outcome). ${ }^{74}$ The ISI is a 7-item questionnaire that assesses the frequency and/or severity of insomnia symptoms (eg, "rate the current severity of your difficulty falling asleep"; choices range from 0 (none) to very severe (5)), as well as questions regarding the impact of insomnia on daytime functioning (eg, "to what extent do you consider your sleep problem to interfere with your daily functioning") (eg, daytime fatigue, mood, ability to function at work/daily chores, concentration, memory, mood, etc)) currently; choices range from 0 (not interfering at all) to 5 (very much interfering). Total scores on the ISI range from 0 to 28 , with higher scores representing more severe insomnia.

\begin{tabular}{|c|c|c|c|}
\hline Objective sleep & Daily actigraphy & Secondary & $\begin{array}{l}\text { Actiwatch } 2 \text { (Philips Respironics) is a watch-like device that monitors } \\
\text { light and gross motor activity. Data will be analysed by proprietary } \\
\text { software using } 30 \text { s epochs. A validated algorithm estimates the same } \\
\text { variables (secondary outcomes) provided by diaries (except sleep quality). } \\
\text { Participants wear the device } 24 / 7 \text { during each } 2 \text { weeks of assessment, } \\
\text { and } 8 \text { weeks of treatment. }\end{array}$ \\
\hline
\end{tabular}

Polysomnographic (PSG) Secondary sleep

The Comet-PLUS Portable (Natus Neurology) Recording System will be used to conduct a single in-home overnight sleep study at baseline, post-treatment and both follow-ups. Consistent with ambulatory recommendations, ${ }^{75}$ monitoring consists of 10 electroencephalography, 2 EOG and 3 electromyography (EMG) (chin) using standard placements. It also includes respiratory inductance plethysmography (thoracic/ abdominal effort), oximeter (pulse/oxygen saturation), ECG, R/L anterior tibialis EMG, oral-nasal airflow thermocouple and nasal cannula pressure transducer. We require 4 hours of acceptable data (ie, scorable stage/ respiratory events) and follow Sleep Heart Health Study ${ }^{76}$ procedures for training, data management and scoring. PSG provides sleep stage \% (stage 1, 2, 3, rapid eye movement sleep) and absolute values for diary variables (secondary outcomes).

\begin{tabular}{|c|c|c|c|}
\hline \multirow[t]{2}{*}{ Arousal } & $\begin{array}{l}\text { Peripheral Arousal-heart } \\
\text { rate variability (HRV) }\end{array}$ & Primary & $\begin{array}{l}\text { Using Holter monitors, we will obtain } 5 \text { min ECG recordings during rest } \\
\text { in a quiet controlled environment at each assessment. Time and spectral } \\
\text { analysis of the short-term variability of heart rate (HR) will be performed } \\
\text { using Pathfinder (Spacelabs, Seattle, Washington) software to assess the } \\
\text { neural regulation of HR. The time domain indices reflect the beat-to-beat } \\
\text { variability with respect to time. The variables SD of the } \mathrm{N}-\mathrm{N} \text { intervals and } \\
\text { the percentage of } \mathrm{N}-\mathrm{N} \text { intervals that exceed } 50 \mathrm{~ms} \text { will be examined. } \\
\text { The frequency domain indices reflect the underlying rhythms of the } \\
\text { mechanisms modulating HR. High frequency }(0.15-0.4 \mathrm{~Hz}) \text {, low frequency } \\
(0.04-0.15 \mathrm{~Hz} \text { ) and very low frequency (below } 0.04 \mathrm{~Hz}) \text { spectral bands will } \\
\text { be examined. }\end{array}$ \\
\hline & $\begin{array}{l}\text { Global cognitive } \\
\text { arousal-Perceived } \\
\text { Stress Scale (PSS) }\end{array}$ & Primary & $\begin{array}{l}\text { The PSS (primary outcome) is a 10-item questionnaire that asks } \\
\text { participants to appraise their stress level during the past month in } \\
\text { response to several everyday situations (eg, "in the last month how often } \\
\text { have you been able to control irritations in your life?"). Choices range from } \\
0 \text { (never) to } 4 \text { (very often). Higher total scores on the PSS indicate worse } \\
\text { perceived stress. }\end{array}$ \\
\hline
\end{tabular}


Table 3 Continued

\begin{tabular}{llll}
\hline $\begin{array}{l}\text { Outcome } \\
\text { category }\end{array}$ & Measure & Primary/Secondary & Details \\
\hline & $\begin{array}{l}\text { Pain-specific cognitive- } \\
\text { affective arousal- } \\
\text { catastrophising-Pain } \\
\begin{array}{l}\text { Catastrophising Scale } \\
(\mathrm{PCS})^{78}\end{array}\end{array}$ & $\begin{array}{l}\text { The PCS is a 13-item scale that measures the degree (from 0-not at all } \\
\text { to } 4-\text { all the time) to which participants experienced certain thoughts or } \\
\text { feelings during past painful events. Items are scored and total scores on }\end{array}$ \\
& the PCS represent worse pain catastrophising.
\end{tabular}

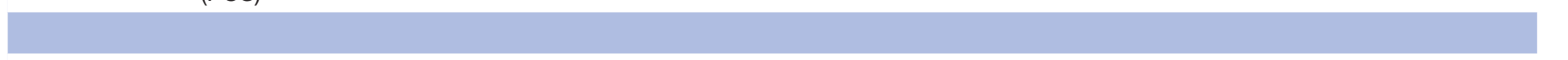

Pain Daily clinical pain- Primary On the daily electronic diaries, participants provide ratings on a 0-100 Electronic Daily Diaries $\quad$ scale regarding their pain intensity $(0-$ no pain sensation, $100-$ most intense pain imaginable) and pain unpleasantness $(0-$ not at all unpleasant, 100-most unpleasant imaginable).

\begin{tabular}{|c|c|c|c|}
\hline & $\begin{array}{l}\text { Subjective pain-McGill } \\
\text { Pain Questionnaire } \\
(\text { MPQ })^{79} 80\end{array}$ & Secondary & $\begin{array}{l}\text { The MPQ assesses participants pain symptoms across } 21 \text { categories. } \\
\text { For each category, participants select the best word that described their } \\
\text { pain. Qualitative responses are coded by numerical value (eg, } 1-3 \text { or } 1-5) \text {, } \\
\text { with higher values representing worse pain in that category. If they do not } \\
\text { experience a specific category of pain, they do not provide a response to } \\
\text { that category. Category scores are summed and total scores could range } \\
\text { from } 0 \text { (no pain) to } 78 \text { (severe pain). }\end{array}$ \\
\hline & $\begin{array}{l}\text { Patient-Centred } \\
\text { Outcomes Questionnaire } \\
(\text { PCOQ })^{81}\end{array}$ & Secondary & $\begin{array}{l}\text { The PCOQ is a 5-item questionnaire that assess on a 0-point to } \\
10 \text {-point scale usual levels of pain, desired levels of pain, what level of } \\
\text { improvement in treatment outcomes they would consider successful, } \\
\text { what level of improvement in treatment outcomes they expect after } \\
\text { treatment, importance of improvement in treatment outcomes. }\end{array}$ \\
\hline & $\begin{array}{l}\text { Pain-related disability- } \\
\text { Pain Disability Inventory } \\
(\text { PDI) }\end{array}$ & Secondary & $\begin{array}{l}\text { The PDI includes } 7 \text {-item questionnaire rated on an } 11 \text {-point scale }(0=\text { no } \\
\text { disability, } 10=\text { total disability) indicating the degree to which chronic pain } \\
\text { interferes with participant functioning in the following areas: family/home } \\
\text { responsibilities, recreation, social activity, occupation, sexual behaviour, } \\
\text { self-care and life-support activity. The seven ratings are summed to } \\
\text { compute a total score }(0-70) \text {, with higher scores indicated worse pain } \\
\text { disability. }\end{array}$ \\
\hline
\end{tabular}

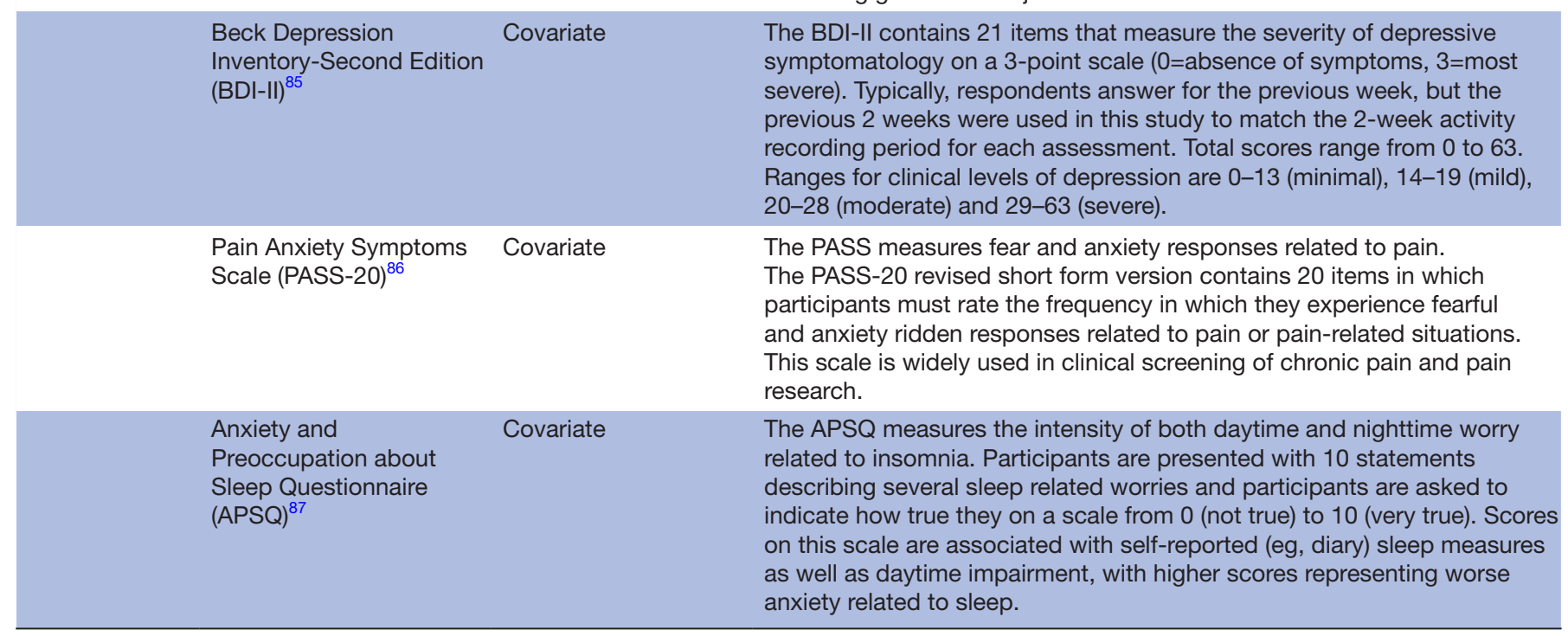

Continued 
Table 3 Continued

\begin{tabular}{lll}
\hline $\begin{array}{l}\text { Outcome } \\
\text { category }\end{array}$ & Measure & Primary/Secondary \\
\hline Neuroimaging & $\begin{array}{l}\text { Neural plasticity and } \\
\text { central sensitisation }\end{array}$ & Primary
\end{tabular}

Details

Three imaging protocols 1) structural MRI (MPRAGE), 2) functional MRI (fMRI) (EPI blood-oxygen-level dependent) and 3) diffusionweighted imaging (DWI), will assess neural plasticity and central sensitisation. Image acquisition. Imaging data will be acquired with Siemens' new MAGNETOM Vida 3T and a 20-channel head-neck coil. The parameters for the 3D-T1-weighted structural scans are: 256 axial slices $\left(0.90 \times 0.89 \times 0.89 \mathrm{~mm}^{3} ; \mathrm{TR}=0.75 \mathrm{~s}, \mathrm{TE}=0.0045 \mathrm{~s}\right.$, flip angle $=750^{\circ}$, matrix $=256 \times 256, F O V=256 \mathrm{~mm}$. T2-gradient $E P I$ sequence for the resting state and fMRI scans will use the following parameters: whole brain, 36-contiguous slices (axial), $3 \mathrm{~mm}^{3}$ isotropic voxels, oriented parallel to the AC-PC plane, $\mathrm{TR}=2.46 \mathrm{~s}$; TE $=30 \mathrm{~ms}$; flip angle $=90^{\circ} ; 76 \times 76$ matrix and 120 volumes. The parameters for the diffusion-weighted scans are: 32 slices, $1 \times 1 \times 3.25 \mathrm{~mm}^{2}, \mathrm{TR}=3.6 \mathrm{~s}, \mathrm{TE}=0.064 \mathrm{~s}$, flip angle $=90^{\circ}$, directions $=6$. The sequence of scan acquisition is: Localizer, gradient field map, 3D anat, resting state ( $\mathrm{x} 2, \sim 5 \mathrm{~min}), \mathrm{fMRI}$ experimental pain scans $(\mathrm{x} 3, \sim 25$ $\mathrm{min})$, DWI ( $\sim 12 \mathrm{~min})$. During the resting state scans, subjects are told to relax, limit movement and try not to fall asleep.

In preparation for the experimental pain scan, participants will first undergo quantitative sensory testing (QST) calibration trials outside of the scanner, in order to determine individual pain tolerance and to ensure that experienced pain intensity is equal in both treatment groups at baseline. A computer-controlled Medoc Pain and Sensory Evaluation System (Pathway Model ATS, Medoc Advanced Medical Systems, Durham, North Carolina) will be used to deliver thermal stimuli. QST calibration uses a series of calibration trials (CTs), to identify their pain tolerance temperature, which will be used during their experimental pain scanning session. The CTs start at $43^{\circ} \mathrm{C}$ and increase by $1^{\circ} \mathrm{C}$ until their tolerance, or $51^{\circ} \mathrm{C}$ is reached, whichever comes first. Subjects will sit in a chair, remove their shoes and socks and extend their feet outward. A researcher will wipe the bottom of their foot with an alcohol pad, after which a contact heat thermode will be placed on the plantar surface of the foot. Each stimulus cycle is initiated by the experimenter via key press. After each stimulus, subjects will describe the sensation (pain/not painful) and rate its pain intensity on a scale from $0-$ no pain to 100 -worst pain imaginable. Once the ratings and interstimulus interval have finished, the cycle will be repeated until their tolerance temperature is identified (ie, the lowest temperature with a pain intensity rating of $\geq 65$ ). This will be the temperature that will be used during their scanning sessions.

During each 5 min experimental pain scan, thermal stimuli will be delivered with an MR compatible, computer-controlled, CHEPS Pathway system, which is a peltier-element-based stimulator, and is capable of producing stimuli across a range of temperatures $\left(33^{\circ} \mathrm{C}-51^{\circ} \mathrm{C}\right)$. The start of each scan will begin with the thermode at ambient temperature for 30 $\mathrm{s}$ and then 16 cycles of the following: $12 \mathrm{~s}$ at ambient temperature, then in $<2 \mathrm{~s}$ the temperature will steadily increase (ramp) until reaching their pain temperature (determined by the calibration trials), and remain at that temperature (hold) for $5 \mathrm{~s}$, followed by a variable inter stimulus interval of 12-20 s. Following the 16th cycle, the scan proceeds for another $30 \mathrm{~s}$ with the thermode at ambient temperature.

${ }^{*}$ Given that our previous clinical trial ${ }^{20}$ evaluating CBT-I relative to a waitlist control on sleep and pain and arousal outcomes found large effect sizes for CBT-I-related improvement in DBAS-assessed cognitive-affective arousal related to sleep, we used the same measure in this trial as well. However, another important index of presleep arousal (including somatic and cognitive arousal) could be captured by using the Pre-Sleep Arousal Scale (PSAS). ${ }^{88}$ Thus, we will consider using the PSAS in future trials.

AC-PC, anterior commissure-posterior commissure ; DWI, diffusion weighted imaging; EOG, electrooculography; EPI, echo planar imaging ; ET, echo time; FOV, field of view; TR, repitition time.

usage). Any variables that are significantly different between groups will be entered in all analyses. We will also include age and education in all analyses as necessary.

Evaluations of aims

Testing of aim 1

To examine the effects of CBT-I on arousal, sleep and pain in patients with fibromyalgia and insomnia, we will use a 2-level MLM. The first level will be the repeated measure over time nested within the second level which is the person-level data. Group (CBT-I, SH) will capture the between-subjects variability, while time (baseline, post-treatment, 6 months, 12 months) will capture withinsubject variability. Based on a priori hypotheses, separate MLMs will be conducted for each sleep, arousal and pain 
Table 4 Schedule of outcome measures

\begin{tabular}{|c|c|c|c|c|c|}
\hline Assessment period & Base & Tx & Post & Boosters & FUs \\
\hline Weeks & 2 & 8 & 2 & 2 & 2 \\
\hline Telephone and clinical interviews, consent, MMSE & $\mathrm{X}$ & & & & \\
\hline Electronic daily diaries & $x$ & $x$ & $\mathrm{X}$ & $x$ & $x$ \\
\hline
\end{tabular}

APSQ, Anxiety and Preoccupation about Sleep Questionnaire; BDI-II, Beck Depression Inventory-Second Edition; DBAS, Dysfunctional Beliefs about Sleep Scale; HRV, heart rate variability; ISI, Insomnia Severity Index; MMSE, Mini Mental State Examination; MPQ, McGill Pain Questionnaire; PASS-20, Pain Anxiety Symptoms Scale; PCS, Pain Catastrophising Scale; PDI, Pain Disability Inventory; PSG, polysomnography; PSS, Pain Severity Scale; RH, Ramp and Hold; STAI, State Trait Anxiety Inventory.

outcome. Planned contrasts will be conducted consistent with a priori hypotheses. Bonferroni-adjusted p values will control family-wise error (FWE). Using an MLM approach allows us to compare group means like in a RM ANOVA, and make comparisons at the individual level. Using an MLM approach, we can answer questions such as: do participants differ at specific time points on the outcome in terms of treatment, do slopes differ in terms of treatment or across participants, do specific time points vary among individuals. MLM allows for comparison of individual trajectories and comparisons between participants.

Clinical significance will also be evaluated for insomnia and pain intensity. Because there are no established clinical significance guidelines for insomnia, participants will be classified as no longer meeting trial criteria for difficulties initiating and maintaining sleep (ie, self-reported SOL or WASO $>30 \mathrm{~min}$ on 3 or more days out of 14) at posttreatment, 6 months follow-up and 12 months follow-up. We will also compare responders (those who no longer meet criteria for insomnia) versus non-responders (those who still meet criteria for insomnia) on all outcomes using independent sample t-tests. In terms of pain, participants will be classified as moderately and substantially improved (pain intensity decreases of $30 \%$ and $50 \%$, respectively) based on provisional benchmarks recommended for determining clinically important differences in pain intensity in clinical trials by the Initiative on Methods, Measurement, and Pain Assessment in Clinical Trials Consensus Panel. ${ }^{60}$ These improvement benchmarks will be examined for both morning and evening pain intensity. Group differences will analysed using $\chi^{2}$ test.

\section{Testing of aim 2 \\ Resting state}

To better understand the effects of treatment on basal brain activity, we will characterise the changes in RS data associated with behavioural changes over time. Towards this end, we will use Group ICA of fMRI Toolbox (GIFT) to perform independent component analyses (ICA) of the RS data. This procedure will decompose the data into discrete components, each representing the unique time course of the brain regions associated with that component (ie, a unique spatial-temporal map). This analytic approach will allow us to identify common (across all groups) and group-specific temporal-spatial ICAs representing the DMN, its subnetworks, pain-related networks, those involved with affective processing and others (eg, sensorimotor networks, saliency network, frontoparietal executive networks, etc). Once identified, GIFT will then be used to test for changes in the effective connectivity and functional coherence among these networks and

Table 5 Study timeline

\begin{tabular}{|c|c|c|c|c|c|c|c|c|c|c|}
\hline Project year $\rightarrow$ & & 1 & & 2 & & 3 & & 4 & & 5 \\
\hline Half $\rightarrow$ & 1 & 2 & 1 & 2 & 1 & 2 & 1 & 2 & 1 & 2 \\
\hline $\begin{array}{l}\text { 1. Develop manual of operating procedures. Register with } \\
\text { clinicaltrials.gov. Publish trial protocol. Develop SH. Train } \\
\text { therapists and assessor. }\end{array}$ & & & & & & & & & & \\
\hline 2. Recruit, collect baseline, deliver treatment & & & & & & & & & & \\
\hline 3. Collect post-treatment assessment & & & & & & & & & & \\
\hline 5. Offer/Provide CBT-I to SH controls & & & & & & & & & & \\
\hline $\begin{array}{l}\text { 6. Final data analysis and dissemination (continues after } \\
\text { grant ends); final report }\end{array}$ & & & & & & & & & & \\
\hline
\end{tabular}

CBT-I, cognitive behavioural treatment for insomnia; $\mathrm{SH}$, sleep hygiene. 
their component brain regions. Then the influence of covariates can then be added to the analyses to examine their influence on specific nodes and overall functional coherence of the network. We will also compare the component representative of the DMN (ie, a specific spatial-temporal map) from each group with a studyspecific DMN map to calculate group-specific differences. By comparing these differences with a standardised DMN template (of healthy controls that is included in GIFT), we can make statistical inferences about grouprelated differences and treatment-related changes over time. Although the standardised template of the DMN included with GIFT is widely used, we acknowledge the limitations of using this template because it is based on healthy individuals. ${ }^{61}$ However, the fact that this template is based on a healthy population, valid inferences about deviations from a normal DMN in a clinical population are possible.

Using the approach outlined above, we anticipate that the component best representing the DMN in both groups will include most, if not all of the following Brodmann areas: BA 11—orbitofrontal area (orbital and rectus gyri), BA 32-dorsal anterior cingulate cortex, BA 9-dorsolateral prefrontal cortex, BA 10-anterior prefrontal cortex (most rostral part of superior and middle frontal gyri), BA 47—orbital part of inferior frontal gyrus, BAs 23 and 31 the ventral and dorsal aspects of the posterior cingulate cortex, BA 39-angular gyrus, BA 40-supramarginal gyrus, BA 37-fusiform gyrus and BAs 30 and 36 of the parahippocampal gyrus. Because participants have chronic pain we expect overlapping pain-related regions to be included (eg, BAs 40, 30, 31). However, given the tonic nature of chronic pain, we expect the DMN might also involve additional pain-related brain regions such as: BAs 4-primary motor cortex, 6-premotor cortex, 16insular cortex, and 46-dorsolateral prefrontal cortex.

\section{Functional MRI}

Using a flexible analytical approach involving MLM and random effects general linear models, we will test for group differences in reported pain and associated painrelated patterns of activity and how those results vary as a function of treatment response and time. To clarify treatment-related changes to painful stimuli, we will identify brain regions of interest (ROIs) wherein the stimuli are significantly convolved with a haemodynamic response function (HRF). When identifying potential ROIs, a combination of criteria are used to guard against type I errors. These criteria are: (A) $\mathrm{p}$ value $\leq 0.05$, using the false discovery rate and FWE corrections; (B) a spatial extent of $50+$ contiguous voxels and a minimum volume of $100 \mu \mathrm{L}$ and $(\mathrm{C})$ the centre of mass gravity/peak voxel in a targeted region. Because all of the imaging data will be in standardised MNI (Montreal Neurological Institute) space, the coordinates of targeted regions will be checked against the standardised Wake Forest Pick Atlas. The combination of these criteria establish an image-wise $p$ value of 0.00002 and an effective pixel-wise alpha of $\mathrm{p} \leq 0.0002{ }^{62}$ This approach will allow us to include additional criteria such as small volume corrections during analyses which may also include area under the curve, growth curve modelling and cluster analyses may also be used to test for group-related differences, over time, in HRF characteristics relative to treatment response and the predictive ability of outcome measures. With the aforementioned analytical approach, we anticipate to identify pain-related activity among typical pain-related brain regions such as: the thalamus, supplementary motor area, primary and secondary somatosensory cortices, anterior and posterior insula, dorsal anterior cingulate cortex and the dorsolateral prefrontal cortex. We hypothesise that these, and other pain-related, regions will be identified at baseline, and that they will be sensitive to treatment effects and changes in other behavioural outcome measures (eg, sleep measures, pain, arousal, etc) over time.

\section{Testing of aim 3}

Structural MRI

To study CBT-I's long-term effect on structural characteristics of pain-related brain regions, we will use the FSL tissue segmentation pipeline for analysis of cortical ribbon changes. The FreeSurfer Longitudinal Processing pipeline is highly specialised to provide unbiased results about longitudinal changes using common and withinsubject templates, allowing for significant increases in reliability and statistical power. ${ }^{63}$ The pipeline accounts for inherent autocorrelations in the data due to repeated sampling allowing us to assess changes among outcome measures (eg, arousal, sleep, pain and grey matter thickness, in ROIs) within/between groups at each interval and longitudinally. Based on the literature ${ }^{39} 40$ 64-67 and our previous results, we anticipate finding significant differences between the CBT-I and SH groups among the somatosensory cortices, cingulate cortices (anterior, mid-dorsal), dorsolateral prefrontal cortex, frontal gyri (superior, middle, inferior), insula (anterior, posterior), temporal gyri, thalamus and periaqueductal grey and their relationship to additional outcome variables. Other behavioural and/or outcome measures refers to possibility of using any other information collected about the participants (eg, sleep measures, pain, arousal, etc).

\section{Diffusion weighted imaging}

The diffusion weighted images (DWI) will be processed via FMRIB's Diffusion Toolbox (FDT) to examine white matter characteristics DWI. DWI measures the diffusion of water across cell membranes in three-dimensional. Because of this, the directionality of the diffusion (anisotropy) can be determined. The FDT pipeline will estimate the apparent diffusion coefficient (ADC-amount of diffusion possible independent of direction) and fractional anisotropy (FA—an index (0 (isotropic diffusion) -1 (diffusion along one vector)) at the individual and group levels. Higher values of FA and reduced ADC represent increased complexity of brain tissue ${ }^{6869}$ Higher values of FA and reduced ADC represent increased complexity of 
brain tissue. ${ }^{6869}$ We will map white matter tracts and model connections among brain regions with probabilistic tractography. ${ }^{4470}$ As this will be a novel contribution to the field, we anticipate potential changes for CBT-I but not $\mathrm{SH}$ in FA and ADC along the prefronto-subcortical dorsolateral-prefrontal and anterior cingulate-prefrontal pathways.

\section{Testing of aim 4}

Finally, to examine the mediating impact of arousal, sleep, and CS on the effects of CBT-I on pain (aim 4), we will use 4-wave cross-lagged path analysis. Controlling for baseline, autoregressions and reciprocal effects among sleep, arousal (HRV, cognitive arousal, stress) and CS (neural factors), we will examine whether CBT-I continues to predict improved sleep and decreased arousal and CS at 6 months, and then predicts pain at 12 months. Mediation effects of arousal, CS and improved sleep of impact of CBT-I on pain outcomes at 12 months will be estimated. We will evaluate whether mediation effects remain significant after controlling for global and pain-specific and sleep-specific cognitive-affective variables.

\section{Patient and public involvement}

Patients and public are not involved in any of the following study procedures: development of research questions and outcome measures, study design, participant recruitment, plan for results dissemination, assessment of burden of intervention.

\section{ETHICS AND DISSEMINATION}

All study procedures were approved by the Institutional Review Board at the University of Missouri on 11 July 2018. An independent four-member Safety Monitoring Committee (SMC) was assembled in January 2019 to oversee the study. Members include those with expertise in fibromyalgia, chronic insomnia, sleep medicine and CBT. All SMC members attested that they have no conflicts of interest. The SMC met once via conference call at the beginning of the study to review the study protocol, Manual of Operating Procedures, Informed Consent Form and monitoring plan with emphasis on data integrity and patient safety issues. Following this initial meeting, the SMC will meet every 6 months to review progress reports prepared by the team biostatistician. Those SMC reports will include interim statistical analyses. Any changes to these procedures that are recommended by the SMC will be adopted. The SMC will review adverse events and monitor study results, focusing on efficacy, recruitment progress, randomisation, compliance, retention, protocol adherence, operating procedures, forms completion, intervention effects, participant safety and minority inclusion. The PI will also submit annual reports to the funding agency.

Results from this trial will be presenting at national conferences, including the Associated Professional Sleep Societies (or SLEEP) and the American Pain Society, in the final year of the project. Dissemination will also occur through the submission of a primary article on the outcomes, a second article focusing on the functional neural changes (ie, RS and fMRI results), a third article focusing on the structural neural changes and a fourth focusing on outcome mediation. The treatment materials will be shared electronically and will be widely available to clinicians.

\section{Author affiliations}

${ }^{1}$ Department of Psychiatry, University of Missouri System, Columbia, Missouri, USA ${ }^{2}$ Departments of Psychiatry and Psychological Sciences, University of Missouri, Columbia, Missouri, USA

${ }^{3}$ Departments of Physical Therapy and Psychological Sciences, University of Missouri, Columbia, Missouri, USA

${ }^{4}$ Department of Health Management and Informatics, University of Missouri, Columbia, Missouri, USA

${ }^{5}$ Department of Neurology, University of Missouri, Columbia, Missouri, USA ${ }^{6}$ Division of Immunology and Rheumatology, University of Missouri, Columbia, Missouri, USA

${ }^{7}$ Department of Rheumatology, University of Florida, Gainesville, Florida, USA ${ }^{8}$ Department of Clinical and Health Psychology, University of Florida, Gainesville, Florida, USA

Acknowledgements The authors would like to thank the ongoing contributions and support from study participants, study staff (research assistants, study coordinator and other site staff) responsible for trial setup, participant recruitment, data collection and data management.

Contributors All authors made substantial contributions to the concept and design of the study. CM drafted initial protocol, with input from all authors. JC, RS, MR drafted MRI protocol. CD drafted statistical analysis plan. CM, PS and CS drafted screening procedures. CM and AFC drafted the manuscript. All authors revised the manuscript.

Funding This work is supported by the National Institute of Nursing Research (NINR) at the National Institute of Health (NIH), grant number NR017168.

Disclaimer The study sponsor was not actively responsible or involved in the study design and will have no involvement in collection, management, analysis or interpretation of data. The sponsor will have no involvement in future manuscript preparation and decision to submit for publication. This research was done without patient involvement. Patients were not invited to comment on the study design and were not consulted to develop patient relevant outcomes. Patients were not invited to contribute to the writing or editing of this document for readability or accuracy.

Competing interests None declared.

Patient and public involvement Patients and/or the public were not involved in the design, or conduct, or reporting, or dissemination plans of this research.

Patient consent for publication Not required.

Provenance and peer review Not commissioned; externally peer reviewed.

Open access This is an open access article distributed in accordance with the Creative Commons Attribution Non Commercial (CC BY-NC 4.0) license, which permits others to distribute, remix, adapt, build upon this work non-commercially, and license their derivative works on different terms, provided the original work is properly cited, appropriate credit is given, any changes made indicated, and the use is non-commercial. See: http://creativecommons.org/licenses/by-nc/4.0/.

\section{ORCID iDs}

Christina S McCrae http://orcid.org/0000-0003-4313-686

Ashley F Curtis http://orcid.org/0000-0002-2311-5674

\section{REFERENCES}

1 Gaskin DJ, Richard P. The economic costs of pain in the United States. J Pain 2012;13:715-24.

2 Andersson $\mathrm{HI}$, Ejlertsson G, Leden I, et al. Impact of chronic pain on health care seeking, self care, and medication. results from a population-based Swedish study. J Epidemiol Community Health 1999;53:503-9. 
3 Association AP. Diagnostic and statistical manual of mental disorders (DSM-5®). American Psychiatric Pub, 2013.

4 Taylor DJ, Mallory LJ, Lichstein KL, et al. Comorbidity of chronic insomnia with medical problems. Sleep 2007;30:213-8.

5 Finan PH, Goodin BR, Smith MT. The association of sleep and pain: an update and a path forward. J Pain 2013;14:1539-52.

6 Harding SM. Sleep in fibromyalgia patients: subjective and objective findings. Am J Med Sci 1998;315:367-76.

7 Moldofsky H. Nonrestorative sleep and symptoms after a febrile illness in patients with fibrositis and chronic fatigue syndromes. $J$ Rheumatol Suppl 1989;19:150-3.

8 Affleck G, Urrows S, Tennen $\mathrm{H}$, et al. Sequential daily relations of sleep, pain intensity, and attention to pain among women with fibromyalgia. Pain 1996;68:363-8.

9 Horne JA, Shackell BS. Alpha-like EEG activity in non-REM sleep and the fibromyalgia (fibrositis) syndrome. Electroencephalogr Clin Neurophysiol 1991;79:271-6.

10 Shaver JL, Lentz M, Landis CA, et al. Sleep, psychological distress, and stress arousal in women with fibromyalgia. Res Nurs Health 1997:20:247-57.

11 Branco J, Atalaia A, Paiva T. Sleep cycles and alpha-delta sleep in fibromyalgia syndrome. J Rheumatol 1994;21:1113-7.

12 Shapiro CM, Devins GM, Hussain MR. Abc of sleep disorders. sleep problems in patients with medical illness. BMJ 1993;306:1532-5.

13 Jennum P, Drewes AM, Andreasen A, et al. Sleep and other symptoms in primary fibromyalgia and in healthy controls. $J$ Rheumatol 1993;20:1756-9.

14 Theadom A, Cropley M, Humphrey K-L. Exploring the role of sleep and coping in quality of life in fibromyalgia. $J$ Psychosom Res 2007;62:145-51.

15 Wolfe F, Smythe HA, Yunus MB, et al. The American College of rheumatology 1990 criteria for the classification of fibromyalgia. Report of the multicenter criteria Committee. Arthritis Rheum 1990;33:160-72.

16 Wolfe F, Clauw DJ, Fitzcharles M-A, et al. The American College of rheumatology preliminary diagnostic criteria for fibromyalgia and measurement of symptom severity. Arthritis Care Res 2010;62:600-10.

17 Morin CM, Hauri PJ, Espie CA, et al. Nonpharmacologic treatment of chronic insomnia. An American Academy of sleep medicine review. Sleep 1999;22:1134-56.

18 Burgmer M, Gaubitz M, Konrad C, et al. Decreased gray matter volumes in the cingulo-frontal cortex and the amygdala in patients with fibromyalgia. Psychosom Med 2009;71:566-73.

19 Smith MT, Quartana PJ, Okonkwo RM, et al. Mechanisms by which sleep disturbance contributes to osteoarthritis pain: a conceptual model. Curr Pain Headache Rep 2009;13:447-54.

20 McCrae CS, Williams J, Roditi D, et al. Cognitive behavioral treatments for insomnia and pain in adults with comorbid chronic insomnia and fibromyalgia: clinical outcomes from the spin randomized controlled trial. Sleep 2019;42. doi:10.1093/sleep/ zsy234. [Epub ahead of print: 01 Mar 2019].

21 Staud R, Vierck CJ, Cannon RL, et al. Abnormal sensitization and temporal summation of second pain (wind-up) in patients with fibromyalgia syndrome. Pain 2001;91:165-75.

22 Staud R, Robinson ME, Vierck CJ, et al. Ratings of experimental pain and pain-related negative affect predict clinical pain in patients with fibromyalgia syndrome. Pain 2003;105:215-22.

23 Eriksen HR, Ursin H. Sensitization and subjective health complaints. Scand J Psychol 2002;43:189-96.

24 Harvey AG. A cognitive model of insomnia. Behav Res Ther 2002:40:869-93.

25 Riemann D, Spiegelhalder K, Feige B, et al. The hyperarousal model of insomnia: a review of the concept and its evidence. Sleep Med Rev 2010;14:19-31.

26 Farina B, Dittoni S, Colicchio S, et al. Heart rate and heart rate variability modification in chronic insomnia patients. Behav Sleep Med 2014;12:290-306.

27 Bonnet MH, Arand DL. Heart rate variability in insomniacs and matched normal sleepers. Psychosom Med 1998;60:610-5.

28 Spiegelhalder K, Fuchs L, Ladwig J, et al. Heart rate and heart rate variability in subjectively reported insomnia. $J$ Sleep Res 2011:20:137-45.

29 Jarrin $\mathrm{DC}$, Chen IY, Ivers $\mathrm{H}$, et al. Nocturnal heart rate variability in patients treated with cognitive-behavioral therapy for insomnia. Health Psychol 2016;35:638-41.

30 Olausson H, Ha B, Duncan GH, et al. Cortical activation by tactile and painful stimuli in hemispherectomized patients. Brain 2001;124:916-27.

31 Stein BE, Price DD, Gazzaniga MS. Pain perception in a man with total corpus callosum transection. Pain 1989;38:51-6.
32 Craggs JG, Price DD, Verne GN, et al. Functional brain interactions that serve cognitive-affective processing during pain and placebo analgesia. Neuroimage 2007;38:720-9.

33 Apkarian AV, Neugebauer V, Koob G, et al. Neural mechanisms of pain and alcohol dependence. Pharmacol Biochem Behav 2013;112:34-41.

34 Moulton EA, Keaser ML, Gullapalli RP, et al. Regional intensive and temporal patterns of functional MRI activation distinguishing noxious and innocuous contact heat. J Neurophysiol 2005;93:2183-93.

35 Garcia-Larrea L, Peyron R. Pain matrices and neuropathic pain matrices: a review. Pain 2013;154:S29-43.

36 lannetti GD, Mouraux A. From the neuromatrix to the pain matrix (and back). Exp Brain Res 2010;205:1-12.

37 Price DD. Psychological and neural mechanisms of the affective dimension of pain. Science 2000;288:1769-72.

38 Ursin H, Eriksen HR. Sensitization, subjective health complaints, and sustained arousal. Ann N Y Acad Sci 2001;933:119-29.

39 Staud R, Craggs JG, Robinson ME, et al. Brain activity related to temporal summation of C-fiber evoked pain. Pain 2007;129:130-42.

40 Staud R, Craggs JG, Perlstein WM, et al. Brain activity associated with slow temporal summation of C-fiber evoked pain in fibromyalgia patients and healthy controls. Eur $\mathrm{J}$ Pain 2008:12:1078-89.

41 Craggs JG, Price DD, Perlstein WM, et al. The dynamic mechanisms of placebo induced analgesia: evidence of sustained and transient regional involvement. Pain 2008;139:660-9.

42 Robinson ME, Craggs JG, Price DD, et al. Gray matter volumes of pain-related brain areas are decreased in fibromyalgia syndrome. $J$ Pain 2011;12:436-43.

43 Kuchinad A, Schweinhardt P, Seminowicz DA, et al. Accelerated brain gray matter loss in fibromyalgia patients: premature aging of the brain? J Neurosci 2007;27:4004-7.

44 Lutz J, Jäger L, de Quervain D, et al. White and gray matter abnormalities in the brain of patients with fibromyalgia: a diffusion-tensor and volumetric imaging study. Arthritis Rheum 2008:58:3960-9.

45 Altena E, Vrenken H, Van Der Werf YD, et al. Reduced orbitofrontal and parietal gray matter in chronic insomnia: a voxel-based morphometric study. Biol Psychiatry 2010;67:182-5.

46 Riemann D, Voderholzer U, Spiegelhalder K, et al. Chronic insomnia and MRI-measured hippocampal volumes: a pilot study. Sleep 2007;30:955-8

47 Cifre I, Sitges C, Fraiman D, et al. Disrupted functional connectivity of the pain network in fibromyalgia. Psychosom Med 2012;74:55-62.

48 Napadow V, LaCount L, Park K, et al. Intrinsic brain connectivity in fibromyalgia is associated with chronic pain intensity. Arthritis Rheum 2010;62:2545-55.

49 Altena E, Van Der Werf YD, Sanz-Arigita EJ, et al. Prefrontal hypoactivation and recovery in insomnia. Sleep 2008;31:1271-6.

50 Huang Z, Liang P, Jia X, et al. Abnormal amygdala connectivity in patients with primary insomnia: evidence from resting state fMRI. Eur J Radiol 2012;81:1288-95.

51 Morin CM, Colecchi C, Stone J, et al. Behavioral and pharmacological therapies for late-life insomnia: a randomized controlled trial. JAMA 1999;281:991-9.

52 Tang NKY, Lereya ST, Boulton $\mathrm{H}$, et al. Nonpharmacological treatments of insomnia for long-term painful conditions: a systematic review and meta-analysis of patient-reported outcomes in randomized controlled trials. Sleep 2015;38:1751-64.

53 Martínez MP, Miró E, Sánchez Al, et al. Cognitive-behavioral therapy for insomnia and sleep hygiene in fibromyalgia: a randomized controlled trial. J Behav Med 2014;37:683-97.

54 Edinger JD, Wohlgemuth WK, Krystal AD, et al. Behavioral insomnia therapy for fibromyalgia patients: a randomized clinical trial. Arch Intern Med 2005;165:2527-35.

55 McCrae CS, Mundt JM, Curtis AF, et al. Gray matter changes following cognitive behavioral therapy for patients with comorbid fibromyalgia and insomnia: a pilot study. J Clin Sleep Med 2018;14:1595-603.

56 Friedman LM, Furberg C, DeMets DL, et al. Fundamentals of clinical trials. Springer, 2010

57 Lichstein KL, Riedel BW, Grieve R. Fair tests of clinical trials: a treatment implementation model. Adv Behav Res Ther 1994;16:1-29.

58 Faul F, Erdfelder E, Buchner A, et al. Statistical power analyses using G*Power 3.1: tests for correlation and regression analyses. Behav Res Methods 2009;41:1149-60.

59 Kenny DA, Judd CM. Power anomalies in testing mediation. Psychol Sci 2014:25:334-9.

60 Dworkin RH, Turk DC, Wyrwich KW, et al. Interpreting the clinical importance of treatment outcomes in chronic pain clinical trials: IMMPACT recommendations. J Pain 2008:9:105-21. 
61 Franco AR, Pritchard A, Calhoun VD, et al. Interrater and intermethod reliability of default mode network selection. Hum Brain Mapp 2009;30:2293-303.

62 Forman SD, Cohen JD, Fitzgerald M, et al. Improved assessment of significant activation in functional magnetic resonance imaging (fMRI): use of a cluster-size threshold. Magn Reson Med 1995;33:636-47.

63 Reuter M, Schmansky NJ, Rosas HD, et al. Within-subject template estimation for unbiased longitudinal image analysis. Neuroimage 2012;61:1402-18.

64 Vatthauer KE, Craggs JG, Robinson ME, et al. Sleep is associated with task-negative brain activity in fibromyalgia participants with comorbid chronic insomnia. J Pain Res 2015;8:819.

65 Craggs JG, Staud R, Robinson ME, et al. Effective connectivity among brain regions associated with slow temporal summation of C-fiber-evoked pain in fibromyalgia patients and healthy controls. $J$ Pain 2012;13:390-400.

66 Craggs J, Robinson M, Price D, et al. Structural and functional brain changes in fibromyalgia: investigation of potential mechanisms associated with central sensitization in chronic pain. $J$ Pain 2010;11:S32.

67 Boissoneault J, Vatthauer K, O'Shea A, O'Shea A, et al. Low-tomoderate alcohol consumption is associated with hippocampal volume in fibromyalgia and insomnia. Behav Sleep Med 2017;15:438-50.

68 Behrens TEJ, Berg HJ, Jbabdi S, et al. Probabilistic diffusion tractography with multiple fibre orientations: what can we gain? Neuroimage 2007;34:144-55.

69 Behrens TEJ, Johansen-Berg H, Woolrich MW, et al. Non-Invasive mapping of connections between human thalamus and cortex using diffusion imaging. Nat Neurosci 2003;6:750-7.

70 Behrens TEJ, Woolrich MW, Jenkinson M, et al. Characterization and propagation of uncertainty in diffusion-weighted MR imaging. Magn Reson Med 2003;50:1077-88.

71 Duerden EG, Albanese M-C. Localization of pain-related brain activation: a meta-analysis of neuroimaging data. Hum Brain Mapp 2013;34:109-49.

72 Lichstein KL, Nau SD, Wilson NM, et al. Psychological treatment of hypnotic-dependent insomnia in a primarily older adult sample. Behav Res Ther 2013;51:787-96.

73 Svendsen K, Borchgrevink P, Fredheim O, et al. Choosing the unit of measurement counts: the use of oral morphine equivalents in studies of opioid consumption is a useful addition to defined daily doses. Palliat Med 2011;25:725-32.

74 Morin CM. Insomnia: psychological assessment and management. Guilford Press, 1993

75 Redline S, Sanders MH, Lind BK, Smith Philip L, Kiley James P, et al. Methods for obtaining and analyzing unattended polysomnography data for a multicenter study. sleep heart health research Group. Sleep 1998;21:759-67.

76 Silva GE, Goodwin JL, Sherrill DL, et al. Relationship between reported and measured sleep times: the sleep heart health study (SHHS). J Clin Sleep Med 2007;3:622-30.

77 Cohen S, Kamarck T, Mermelstein R. Perceived stress scale. In: Measuring stress: a guide for health and social scientists, 1994.

78 Sullivan MJL, Bishop SR, Pivik J. The pain catastrophizing scale: development and validation. Psychol Assess 1995;7:524-32.

79 Melzack R. The McGill pain questionnaire: major properties and scoring methods. Pain 1975;1:277-99.

80 Katz J, Melzack R. Measurement of pain. Surg Clin North Am 1999;79:231-52.

81 Robinson ME, Brown JL, George SZ, et al. Multidimensional success criteria and expectations for treatment of chronic pain: the patient perspective. Pain Med 2005;6:336-45.

82 Pollard CA. Preliminary validity study of the pain disability index. Percept Mot Skills 1984;59:974.

83 Tait RC, Pollard CA, Margolis RB, et al. The pain disability index: psychometric and validity data. Arch Phys Med Rehabil 1987;68:438-41.

84 Spielberger CD, Gorsuch RL, Lushene R, et al. State-trait anxiety inventory, form Y. Palo Alto, CA: Consulting Psychologists Press, 1983.

85 Beck AT, Steer RA, Garbin MG. Beck depression inventory. 2 edn. San Antonio, TX: The Psychological Corporation, 1996.

86 McCracken LM, Dhingra L. A short version of the pain anxiety symptoms scale (PASS-20): preliminary development and validity. Pain Res Manag 2002;7:45-50.

87 Tang NKY, Harvey AG. Correcting distorted perception of sleep in insomnia: a novel behavioural experiment? Behav Res Ther 2004;42:27-39.

88 Nicassio PM, Mendlowitz DR, Fussell JJ, et al. The phenomenology of the pre-sleep state: the development of the pre-sleep arousal scale. Behav Res Ther 1985;23:263-71. 